\title{
Prevalence of Multidrug Resistant (MDR) Proteus spp. in Burn Wound Infection of a Tertiary Care Hospital, Rajshahi
}

\author{
Anika Tasnim ${ }^{1}$, Md. Shah Alam ${ }^{2}$, Md. Abdullah Yusuf ${ }^{3}$, Fazlay Akbar Khan ${ }^{4}$, Jannatul Ferdose ${ }^{5}$, \\ Mahmuda Sultana ${ }^{6}$ \\ ${ }^{1}$ Department of Microbiology, Parkview Medical College, Sylhet, Bangladesh \\ ${ }^{2}$ Department of Microbiology, Rajshahi Medical College, Rajshahi, Bangladesh \\ ${ }^{3}$ Department of Microbiology, National Institute of Neurosciences and Hospital, Dhaka, Bangladesh \\ ${ }^{4}$ Department of Orthopedic Surgery, Sylhet MAG Osmani Medical College, Sylhet, Bangladesh \\ ${ }^{5}$ Pathology and Microbiology Department, National Institute of Diseases of Chest and Hospital, Mohakhali, Dhaka, Bangladesh \\ ${ }^{6}$ Department of Anatomy, Parkview Medical College, Sylhet, Bangladesh
}

Email address:

anika_r46@yahoo.com (A. Tasnim),drmdshahalam1963@gmail.com (Md. S. Alam), ayusuf75@yahoo.com (Md. A. Yusuf), fazlayakbarkhan@gmail.com (F. A. Khan),jferdose20@gmail.com (J. Ferdose), mahmudasultana90@gmail.com (M. Sultana)

\section{To cite this article:}

Anika Tasnim, Md. Shah Alam, Md. Abdullah Yusuf, Fazlay Akbar Khan, Jannatul Ferdose, Mahmuda Sultana. Prevalence of Multidrug Resistant (MDR) Proteus spp. in Burn Wound Infection of a Tertiary Care Hospital, Rajshahi. International Journal of Infectious Diseases and Therapy. Vol. 6, No. 2, 2021, pp. 65-68. doi: 10.11648/j.ijidt.20210602.14

Received: April 29, 2021; Accepted: May 27, 2021; Published: June 4, 2021

\begin{abstract}
Background: Multidrug resistant (MDR) bacteria are an emerging public health issue in all over the world especially in developing countries like Bangladesh. Objective: This study was aimed to determine the prevalence of MDR Proteus species in burn wound infected patients admitted in Burn and Plastic Surgery Unit, Rajshahi Medical College Hospital (RMCH). Methodology: This cross sectional study was conducted at Microbiology Department of Rajshahi Medical College, Rajshahi, Bangladesh. A total of 212 wound swabs were collected and processed as per standard protocol from January to December 2016 for a period of one year. The isolation and identification of Proteus species was done by conventional microbiological process and antimicrobial susceptibility was performed by Modified Kirby-Bauer disk diffusion method. The Proteus species was further classified into MDR on the basis of standardised international criteria. Result: Among the 196 isolates from 212 wound swabs, Proteus spp. was the $2^{\text {nd }}$ most frequent isolated organism (23.47\%) following Pseudomonas aeruginosa (34.18\%). Two species of Proteus were identified; Proteus mirabilis (65.22\%) and Proteus vulgaris $(34.78 \%)$. More than $75.0 \%$ Proteus spp. showed resistance to ciprofloxacin and ceftriaxone where meropenem was the highest sensitive drug (>93\%). MDR Proteus spp. were 63.04\% among which Proteus mirabilis and Proteus vulgaris were 60\% \& $68.75 \%$ MDR respectively. Conclusion: This study culminates the high prevalence of MDR Proteus spp. in Burn Unit of RMCH and decreased sensitivity to commonly used antibiotics. Therefore a greater emphasis on prevention of MDR bacterial colonization and antibiotic stewardship are imperative.
\end{abstract}

Keywords: Burn Wound Infection, Multidrug Resistant (MDR), Proteus spp.

\section{Introduction}

Burn wound infection still persists as one of the main source of mortality and morbidity [1]. It provides moist and nourishing surroundings conductive to microbial colonization, proliferation and infection [2]. Burn patients are infected by hospital-acquired bacteria by numerous invasive and noninvasive procedures [1].
Different species of microorganisms are responsible for burn wound infection that varies in their frequency with geographical location. Among the Gram negative bacilli Proteus spp. is one of the culprits. The genus Proteus belongs to the tribe of Proteeae within the Enterobacteriaceae family consisting of Proteus, Providencia and Morganella genera. P.mirabilis, P.vulgaris, P.penneri, and P.myxofaciens are the four species that belongs to Proteus genus. The last one is 
insignificant in infections of human [3].

A variety of opportunistic nosocomial infections are caused by Proteus spp. that includes respiratory tract, ear, nose, skin, burns, and wounds [3]. Proteus mirabilis is the species most frequently recovered from human, particularly from wound infections and urinary tract accounting $90 \%$ of all infections caused by the Proteus spp. [4]. It is also familiar to find Proteus mirabilis bacilli in immunocompromised individuals like infected burn wounds [5].

Nowadays, majority of the bacteria that cause nosocomial burn infection shows resistant to at least one of commonly used antimicrobials [1]. A wide range of variables (biological, pharmacological and societal) are responsible antimicrobial resistance that occurs globally particularly in developing countries like Bangladesh [6]. The excessive and misuse of antibiotics in treating bacterial infections have evolved in the emergence of resistant strains that are difficult to treat [5].

At present the World Health Organization (WHO) has notified MDR bacteria as the most threatened issue that created negative impacts on prevention and treatment of bacterial infections [6]. The International Expert Proposal for Interim Standard Definitions for Acquired Resistance had defined MDR, which entailed non-susceptibility to three (3) or more specified groups of antimicrobials [7].

The significant evolution and rise of multidrug-resistance of numerous bacterial isolates is escalating day by day which has evoked a growing public health issue in the world. MDR Proteus spp. necessitates frequent monitoring of antimicrobial susceptibility pattern aiming to apply appropriate antimicrobial therapy [8]. Therefore, this study was performed to determine the prevalence of MDR Proteus spp. from burn wound infected patients in $\mathrm{RMCH}$, Bangladesh.

\section{Materials and Methods}

This cross sectional study was carried out at Microbiology Department of Rajshahi Medical College (RMC) among burn wound infected patients admitted in Burn and Plastic Surgery Unit of RMCH, Rajshahi, Bangladesh from January to December 2016. Patients who had the history of burn more than 3 days irrespective of age, sex and duration of hospital stay were included in the present study. All relevant information was recorded in a predesigned data sheet.

\subsection{Isolation and Identification of Proteus Species}

The wound swabs were collected aseptically and were processed by standard microbiological methods at Microbiology Department of RMC. Samples were inoculated in Blood agar media, Nutrient agar media and MacConkey's agar media aerobically at $37^{\circ} \mathrm{C}$ for 24 hours. Colonies that were non-lactose fermenting on MacConkey agar and showed swarming on Blood agar were isolated and identified by biochemical tests based on whether they were positive for phenylalanine deaminases production; $\mathrm{H}_{2} \mathrm{~S}$ gas production; and urease reactions. P.vulgaris produces indole which differentiated it from indole negative P.mirabilis.

\subsection{Antimicrobial Susceptibility Test}

Isolated bacteria were tested for antimicrobial susceptibility by modified Kirby-Bauer disk diffusion method using Mueller-Hinton agar media and commercially available antimicrobial disks following the guideline of CLSI 2015 [9]. Gram Negative bacteria were tested for amikacin $(30 \mu \mathrm{g})$, gentamycin $(10 \mu \mathrm{g})$, ciprofloxacin $(5 \mu \mathrm{g})$, cefuroxime $(30 \mu \mathrm{g})$, ceftriaxone $(30 \mu \mathrm{g})$, ceftazidime $(30 \mu \mathrm{g})$, amoxiclav $(20 / 10 \mu \mathrm{g})$ and meropenem $(10 \mu \mathrm{g})$. The isolates were considered as MDR when non-susceptibility to at least one agent in three or more antimicrobial categories [7]. Six frequently used classes of antibiotics were used to analyze MDR.

\section{Result}

Out of 212 wound swabs $89.62 \%$ yielded growth in culture and 196 bacterial species were isolated. Pseudomonas aeruginosa was the predominant organism (34.18\%) followed by Proteus spp., the $2^{\text {nd }}$ highest isolated organism (23.47\%) in burn wound infection (Table 1). Proteus mirabilis and Proteus vulgaris were identified among 46 Proteus spp. Proteus mirabilis was most frequently isolated species $(65.22 \%)$ followed by Proteus vulgaris (34.78\%) (Figure 1).

Table 1. Aerobic bacteria isolated from burn wound infected cases $(N=212)$.

\begin{tabular}{lll}
\hline Isolated organisms & No & Percent \\
\hline Pseudomonas aeruginosa & 67 & 34.18 \\
Proteus spp. & 46 & 23.47 \\
Klebsiella spp. & 29 & 14.80 \\
Escherichia coli & 23 & 11.73 \\
Staphylococcus aureus & 21 & 10.71 \\
Acinetobacter spp. & 04 & 2.04 \\
CoNS & 04 & 2.04 \\
Enterobacter spp. & 02 & 1.03 \\
Total Isolates & 196 & 100 \\
\hline
\end{tabular}

Note: $\mathrm{N}=$ Total sample (wound swab) number.

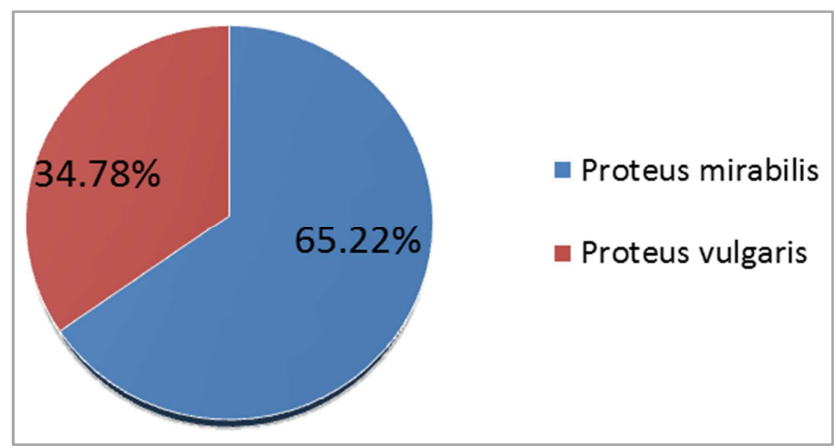

Figure 1. Distribution of isolated Proteus spp.

Distribution of Proteus spp. was analyzed in different age group. Proteus infection was highest in the age group 21-30 years followed by $31-40$ years (Table 2). 
Table 2. Distribution of Proteus spp. in different age group $(n=46)$.

\begin{tabular}{llll}
\hline Age (years) & Proteus mirabilis & Proteus vulgaris & Total no \\
\hline$\leq 10$ & 04 & 01 & 05 \\
$11-20$ & 05 & 02 & 07 \\
$21-30$ & 09 & 07 & 16 \\
$31-40$ & 08 & 03 & 11 \\
$41-50$ & 03 & 01 & 04 \\
$51-60$ & 01 & 00 & 01 \\
$>60$ & 00 & 02 & 02 \\
Total & 30 & 16 & 46 \\
\hline
\end{tabular}

Note: $\mathrm{n}=$ Total number of Proteus spp.

Regarding antimicrobial susceptibility Proteus spp. were highly resistant nearly $>75 \%$ to ciprofloxacin \& ceftriaxone and more than $65 \%$ to gentamycin \& ceftazidime. Meropenem was the highest sensitive drug ( $>90 \%)$ followed by amikacin (>60\%) (Figure 2 ).

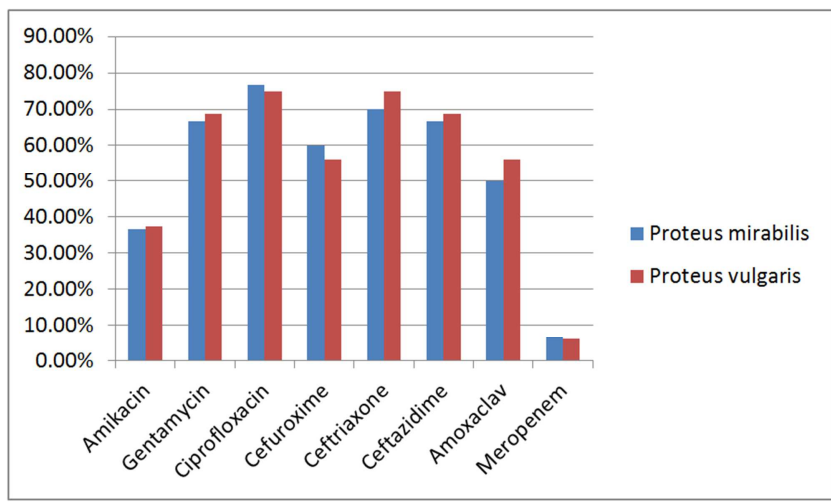

Figure 2. Antimicrobial resistant pattern of Proteus spp.

In this study the prevalence of MDR Proteus spp. was high. More than $60 \%$ of all isolates were found to be MDR among which Proteus mirabilis were 60\% and Proteus vulgaris were $68.75 \%$ (Table 3 ).

Table 3. Multidrug resistant (MDR) Proteus spp.

\begin{tabular}{lll}
\hline Organism & Total no & No of isolates found to be MDR \\
\hline Proteus mirabilis & 30 & $18(60.00 \%)$ \\
Proteus vulgaris & 16 & $11(68.75 \%)$ \\
Total & 46 & $29(63.04 \%)$ \\
\hline
\end{tabular}

Figures in parenthesis represent percentage.

\section{Discussion}

The onset of infectious diseases and drug resistance are very common in developing and densely populated country like Bangladesh [10]. Therefore to manage and control the infection rate, species identification and antimicrobial resistance surveillance is essential.

Out of 196 isolates, Pseudomonas aeruginosa was the most frequent microbial isolates $(34.18 \%)$ in this study. In Bangladesh, Pseudomonas aeruginosa was reported as the predominant organism of burn wound infection in 39\% cases which was in agreement with this study [11]. Proteus spp. the 2nd most frequent bacterial isolate, were $23.47 \%$, which was in accordance with other studies done in Bangladesh and
India $[12,13]$. However, in contrast to this findings, a very lower isolation of Proteus spp. was also reported [14, 15].

In the present study two species of Proteus (Proteus mirabilis and Proteus vulgaris) were identified to be responsible for burn wound infection. Proteus mirabilis was the most commonly isolated species $(65.22 \%)$ and hence accountable for the most of the Proteus infection which was followed by Proteus vulgaris (34.78\%). This finding was consistent with other studies conducted in Ghana [16] and India [9, 17]. According to Mordi and Momoh, a lower isolation of P.mirabilis and P.vulgaris was also reported [4]. The occurrence of burn wound infection in different age group by Proteus mirabilis and Proteus vulgaris was analyzed. The incidence was higher in 21-30 years age group which was quite similar with studies done in India [9].

Antimicrobial susceptibility pattern revealed that Proteus spp. were resistant to commonly used antibiotics like $3^{\text {rd }}$ generation cephalosporin, quinolones etc which are being used indiscriminately on empirical basis for prolong duration of time. In this study Proteus spp. were $>75 \%$ resistant to ciprofloxacin \& ceftriaxone and $>65 \%$ to gentamycin \& ceftazidime. Meropenem was the highest sensitive drug ( $>90 \%)$ followed by amikacin. Previous reports within or outside Bangladesh showed similar findings $[5,12,18]$. Cent percent sensitivity to ciprofloxacin, gentamycin $\&>80 \%$ to ceftriaxone were also reported which was unlike to this study [6, 19].

Proteus spp. showed a high level of MDR (63.04\%). Among them $60 \%$ of Proteus mirabilis and $68.75 \%$ of Proteus vulgaris were MDR which was in agreement with other studies published in India [9, 15], Ghana [16] and in Egypt [1]. The high incidence of MDR Proteus may be due to the inappropriate use of broad spectrum antimicrobial without sensitivity testing. This findings, however, in contrast with Pandey et al., who had reported a very low level of MDR Proteus spp. [17].

\section{Conclusion}

Immense bacterial proliferation and high prevalence of MDR Proteus spp. were observed in the present study. Commonly used $3^{\text {rd }}$ generation cephalosporin and quinolone group of drugs were highly resistant whereas meropenem was the most sensitive drug. This highlights the alarming levels of antimicrobial resistance and need for applying effective antibiotic therapy. Therefore, MDR bacteria should be identified along with their antibiogram by every burn center in order to help clinicians to choose the most suitable antimicrobial therapy for patient benefit as well as to reduce emergence of drug resistant bacteria.

\section{Acknowledgements}

Authors gratefully acknowledge Late Professor A. K. M. Shamsuzzaman, who guided and gave full contribution to this study. We also thankful to the technical support provided by Department of Microbiology of Rajshahi Medical College and Burn and Plastic Surgery Unit of Rajshahi Medical 
College Hospital, Rajshahi, Bangladesh.

\section{References}

[1] Melake NA, Eissaa NA, Keshkb TF, Sleem AS. (2015). Prevalence of multidrug- resistant bacteria isolated from patients with burn infection. Menoufia Medical Journal. 28: 677-684.

[2] Alam MM, Islam MN, Hawlader MDH, Ahmed S, Wahab A, Islam M, KM Roshed Uddin KMR, Hossain A. (2020). Prevalence of multidrug resistance bacterial isolates from infected wound patients in Dhaka, Bangladesh: A crosssectional study. International Journal of Surgery Open. 28 (2021): 56-62.

[3] Al-Bassam WW, Al-Kazaz AK. (2013). The Isolation and Characterization of Proteus mirabilis from Different Clinical Samples. Journal of Biotechnology Research Center. 7 (2): 2430 .

[4] Mordi RM, Momoh MI. (2009). Incidence of Proteus species in wound infections and their sensitivity pattern in the University of Benin Teaching Hospital. African Journal of Biotechnology. 8 (5): 725-730.

[5] Abbas HA, El-Saysed MA, Ganiny AM, Fattah AA. (2018). Antimicrobial Resistance Patterns of Proteus mirabilis isolates from Urinary tract, burn wound and Diabetic foot Infections. Research Journal of Pharmacy and Technology. 11 (1): 249252 .

[6] Gallaher JR, Banda W, Lachiewicz AM, Krysiak R, Cairns BA, Charles AG. (2018). Colonization with Multidrug Resistant Enterobacteriaceae is Associated with Increased Mortality Following Burn Injury in Sub-Saharan Africa. World J Surg. 42 (10): 3089-3096.

[7] Magiorakos AP, Srinivasan A, Carey RB, Carmeli Y, Falagas ME, Giske CG et al. (2012). Multidrug-resistant, extensively drug-resistant and pandrug-resistant bacteria: an international expert proposal for interim standard definitions for acquired resistance. Clin Microbiol Infect Dis. 18: 268-281

[8] Pal N, Sharma N, Sharma R, Hooja S, Maheshwari RK. (2014). Prevalence of Multidrug (MDR) and Extensively Drug Resistant (XDR) Proteus species in a tertiary care hospital, India. Int. J. Curr. Microbiol. App. Sci. 3 (10): 243-252.

[9] CLSI. (2015). Performance Standards for Antimicrobial
Susceptibility Testing; Twenty-Fifth Informational Supplement.

[10] Alam SMS, Kalam MAK, Munna MS, Munshi SK, Noor R. (2014). Isolation of pathogenic microorganisms from burn patients admitted in Dhaka Medical College and Hospital and demonstration of their drug-resistance traits. Asian pacific Journal of Tropical Disease. 4 (5): 402-407

[11] Saha SK, Muazzam N, Begum SA, Chowdhury A, Islam MS, Parveen R. (2011). Study on Time-related Changes in Aerobic Bacterial Pattern of Burn Wound Infection. Faridpur Med Coll J. 6 (1): 41-45.

[12] Begum H, Quamruzzaman M, Talukdar M. (2011). Microbial Isolates from Patients and their Antibiogram at the Tertiary care Burn Unit in Bangladesh. J Bangladesh CollPhys Surg. 29: $62-66$.

[13] Lakshmi N, Koripella R, Manem J, Krishna PB. (2015). Bacteriological profile and Antibiogram of Burn wound infections in a tertiary care hospital. IOSR Journal of Dental and Medical Sciences. 14 (10): 1-4.

[14] Mehta M, Dutta P, Gupta V. (2007). Bacterial isolates from burn wound infections and their antibiograms: A eight-year study. Indian Journal of Plastic Surgery. 40 (1): 25-28.

[15] Perween N, Prakash SK, Bharara T. (2016). Prevalence of Multidrug-Resistant and Extensively Drug-Resistant Proteus, Providencia and Morganella Species in Burn Wound Infection. International Journal of Scientific Study. 3 (11): 154-156.

[16] Feglo PK, Gbedema SY, Quay SNA, Adu-Sarkodie Y, OpokuOkrah C. (2010). Occurance, species distribution and case study at the Komfo Anokye Teaching Hospital (KATH) in Ghana. International Journal of Pharma Sciences and Research (IJPSR). 1 (9): 347-352.

[17] Pandey JK, Narayan A, Tyagi S. (2013). Prevalence of Proteus species in clinical samples, antibiotic sensitivity pattern and ESBL production. International Journal of Current Microbiology and Applied Sciences. 2 (10): 253-261.

[18] Rao SR, Lakshmi LJ, Pavani S, Kawle V, Prakash SJ. (2014). Bacteriological profile, antibiogram of burn wound isolates and detection of MRSA and ESBL production at tertiary care hospital, Hyderabad. World Journal of Pharmacy and Pharmaceutical Sciences. 3 (10): 1691-1698.

[19] Rajbahak S, Shrestha C, Shrestha J, Singh A. (2014). Bacteriological changes of burn wounds with time and their antibiogram. Scientific World. 12 (12): 70-76. 